\title{
Revisão de prontuário como estratégia de ensino-aprendizagem da medicina centrada na pessoa em um ambulatório universitário no município do Rio de Janeiro
}

\author{
Medical record review as a strategy of teaching and learning on patient-centered medicine at a university ambulatory care \\ unit in the municipality of Rio de Janeiro

\section{Revisión de registro médico como estrategia de enseñanza-aprendizaje de la medicina centrada en el paciente en un ambulatorio universitario en el municipio de Rio de Janeiro}

Ana Cláudia Santos Chazan. Universidade do Estado do Rio de Janeiro (UERJ). Rio de Janeiro, RJ, Brasil. anachazan@gmail.com (Autora correspondente) Lia Márcia Cruz da Silveira. Universidade Federal do Rio de Janeiro (UFRJ). Rio de Janeiro, RJ, Brasil. liadasilveira@gmail.com

Cesar Augusto Orazem Favoreto. Universidade do Estado do Rio de Janeiro (UERJ). Rio de Janeiro, RJ, Brasil. cesarfavoreto@globo.com

\section{Resumo}

0 objetivo do presente estudo é relatar a experiência do uso de um roteiro elaborado para orientar a revisão de prontuários em um ambulatório de um hospital universitário no município do Rio de Janeiro. 0 roteiro constava de questões fechadas e abertas abordando os seguintes itens: identificação do paciente, motivo e data da primeira consulta, informações subjetivas, informações objetivas, avaliação das necessidades de saúde do paciente, do plano terapêutico, dos registros, da longitudinalidade do cuidado e conclusões (resumo diagnóstico e terapêutico, proposições para a melhoria dos cuidados e necessidades de aprendizagem). Um prontuário foi escolhido aleatoriamente, copiado e distribuído a seis preceptores voluntários, juntamente com o roteiro. Percebeu-se que, na análise do prontuário, informações importantes de identificação dos usuários e da anamnese estavam ausentes ou desatualizadas. Observou-se concordância em $60 \%$ das respostas às perguntas fechadas. A heterogeneidade foi maior para perguntas abertas referentes à compreensão sobre risco, proteção, vulnerabilidade e necessidades não percebidas pelo usuário. Todos os preceptores consideraram o prontuário incompleto e observaram que os registros, centrados na doença, não eram assinados pelo preceptor. Além disso, foram identificados, como necessidades de aprendizagem, o conhecimento biomédico atualizado sobre as doenças de base do paciente e as habilidades sobre abordagem centrada na pessoa. A revisão do prontuário orientada pelo roteiro permitiu aos preceptores perceberem a necessidade de lerem os registros feitos pelos internos nos prontuários após a discussão dos casos, apontou temas para a educação permanente e os levou a refletir sobre o seu papel na formação profissional dos internos do curso de medicina.

\section{Abstract}

The objective of the present study is to report the experience of the use of a guideline elaborated to guide the review of medical records in an outpatient care unit of a University Hospital in the municipality of Rio de Janeiro. The guideline included open and closed questions regarding patient identification, reason and date of the first appointment, subjective and objective information, evaluation of patients' health needs, therapeutic plan, entries, continuity of care, and conclusions (diagnostic and therapeutic summary, propositions for improvement of care and learning needs). A medical record was randomly selected, copied and distributed to six voluntary preceptors together with the guideline. Through the analysis of the medical records, it was possible to notice that important information for identification and anamnesis of users was missing or not up-to-date. Concordance was observed in $60 \%$ of the closed questions. Heterogeneity was more frequently seen in open questions concerning the understanding about risk, protection, vulnerability, and unrecognized needs of users. All preceptors considered the records incomplete and observed that the entries, which were focused on the diseases, had not been signed by the preceptor. In addition, knowledge regarding patients' diseases and patient-centered clinical methods were identified as learning necessities. Therefore, the guideline-oriented review of the medical records allowed preceptors to recognize the need for reading medical students' notes in patients' records after discussing the cases, pointed out topics for their continuing medical education, and influenced a reflection about their role in the professional development of medical students.
Palavras-chave:

Registros Médicos Atenção Primária à Saúde Assistência Centrada no Paciente Educação Médica

Keywords:

Medical Records Primary Health Care Patient-Centered Care Education, Medical 


\section{Resumen}

El objetivo de este estudio es presentar la experiencia del uso de un guión diseñado para orientar la revisión de los registros médicos en un ambulatorio de un hospital universitario en el municipio de Rio de Janeiro. El guión contenía preguntas cerradas y abiertas que abordaban los siguientes temas: identificación del paciente, causa y fecha de la primera cita, informaciones subjetivas, informaciones objetivas, evaluación de las necesidades de salud del paciente, del plan de tratamiento, de los registros, de la atención longitudinal y las conclusiones (resumen diagnóstico y terapéutico, propuestas para mejorar la atención y las necesidades de aprendizaje). Se seleccionó aleatoriamente un registro médico, copiado y distribuido a seis preceptores voluntarios junto con el guión. En el análisis del registro médico se observó que no aparecían importantes informaciones de identificación de los usuarios y de la anamnesis, 0, si aparecían, estaban desactualizadas. Hubo concordancia en el $60 \%$ de las respuestas a las preguntas cerradas. La heterogeneidad fue mayor para preguntas abiertas respecto a la comprensión del riesgo, la protección, la vulnerabilidad y necesidades desapercibidas para el usuario. Todos los preceptores consideraron incompleto el historial y observaron que los registros, centrados en la enfermedad, no estaban firmados por el preceptor. Además, el conocimiento biomédico actualizado sobre las enfermedades de base del paciente y las habilidades de abordaje centrados en la persona se identificaron como necesidades de aprendizaje. La revisión del historial médico, orientada por el guión, permitió que los preceptores comprendieran la necesidad de lectura de los registros hechos por los estudiantes en los historiales después de la discusión de los casos, indicó temas para su educación permanente y provocó una reflexión en relación a sus roles en la formación profesional de los estudiantes de medicina.
Palabras clave:

Registros Médicos

Atención Primaria de Salud Atención Dirigida al Paciente Educación Médica

\section{Introdução}

O método clínico centrado na pessoa (MCCP) pressupóe mudanças na atitude médica para uma compreensão mais abrangente do processo de saúde e adoecimento das pessoas. Esse método é preconizado pela Medicina de Família e Comunidade (MFC) e fundamenta-se na investigação, de forma equilibrada, tanto da doença, seus "aspectos objetivos", quanto da experiência de estar doente e o contexto de vida da pessoa. ${ }^{1}$

Desse modo, seria possível ao médico de família qualificado reconhecer não apenas os sentimentos e as ideias das pessoas sobre sua condição, mas também identificar possíveis danos funcionais e expectativas dos usuários em relação ao atendimento buscado. ${ }^{2}$

$\mathrm{Na}$ perspectiva dos princípios que orientam este método clínico, a doença não está separada da pessoa e a pessoa não está separada de seu ambiente. O uso apropriado do MCCP requer uma escuta cuidadosa da história e das necessidades da pessoa e uma atenção apurada para possíveis sinais em sua narrativa (verbais ou náo verbais) que favoreçam a compreensão e a condução da situação, além da avaliação das circunstâncias que acompanham o início dos sintomas. ${ }^{3}$

Nesse sentido, é fundamental o investimento na relação médico-paciente/usuário, que se estabelece com base na empatia e no desenvolvimento da confiança mútua, para a construção do vínculo e da aliança terapêutica, sendo necessário ao médico ter consciência de si mesmo e dos aspectos inconscientes que permeiam esta relação, como a transferência e a contratransferência. ${ }^{1,3}$

As disciplinas de Medicina Integral I, II e III, que tratam respectivamente da abordagem comunitária, familiar e da pessoa, foram criadas em 2002, pelo Departamento de Medicina Integral Familiar e Comunitária (DMIF), dentro do processo institucional da Faculdade de Ciências Médicas (FCM) da Universidade do Estado do Rio de Janeiro (UERJ) de adequação do ensino médico às Diretrizes Curriculares Nacionais (DCN) do Curso de Graduação em Medicina ${ }^{4}$ e têm o objetivo de apresentar aos estudantes os atributos da Atenção Primária à Saúde (APS), por meio dos princípios e práticas da MFC. ${ }^{5}$

Desde então, seus conteúdos têm passado por processos avaliativos permanentes para adequação da integração entre teoria e prática e para a aquisição de habilidades e atitudes adequadas ao exercício do (MCCP). Além disso, pretende-se que, ao longo dos três semestres em que as disciplinas são oferecidas, os estudantes avancem na compreensão do Sistema Único de Saúde (SUS) como uma rede de serviços de saúde articulados, em que a qualificação e o compromisso dos profissionais de saúde são fundamentais para a efetivação das políticas públicas.

O Ambulatório de Medicina Integral (AMI) foi criado na década de 1970 com o objetivo de oferecer aos estudantes e residentes do Hospital Universitário Pedro Ernesto (HUPE), um cenário de ensino-aprendizagem de cuidados de atenção primária, voltado para o atendimento das necessidades de saúde das pessoas. ${ }^{6}$

No AMI, os internos (alunos do quinto ano do curso de Medicina) exercem a prática clínica ambulatorial de forma supervisionada por um ano, em um turno semanal de quatro horas, em uma relaçáo de quatro a cinco internos para cada preceptor. Este arranjo organizacional e pedagógico responsabiliza o interno pelos pacientes, permitindo a continuidade na atenção, e possibilita a construção de vínculos e avaliação do projeto terapêutico desenvolvido. 
Deste encontro entre interno-paciente-preceptor emergem experiências que afetam diretamente todos os envolvidos. Os internos, apesar da ansiedade por fazerem diagnósticos de doenças e prescriçôes farmacológicas, são instigados a perceberem as pessoas que estão por detrás dos sinais e sintomas.

Os pacientes a cada ano precisam se readaptar aos "novos médicos", mas se sentem seguros pela referência constante dos preceptores, que, por sua vez, têm a função desafiadora de mediar a relação entre os internos, os pacientes, o conhecimento biomédico e as práticas de cuidado às pessoas.

A dinâmica do rodízio anual dos internos ${ }^{7}$ leva, por um lado, à agregação de diferentes olhares sobre o mesmo usuário e, por outro lado, à descontinuidade progressiva de informaçôes relevantes da história das pessoas, que deixam de constar nos registros subsequentes. Tendo em vista o prontuário como fio condutor da comunicação entre interno-paciente-preceptor a cada ano e entre os internos ao longo dos anos, a revisão periódica dos prontuários se faz necessária como um meio de avaliação e monitoramento da qualidade desta comunicação e do cuidado oferecido aos usuários.

O objetivo deste artigo é apresentar como se deu o processo de construção e avaliação por pares de um roteiro para auxiliar os internos e preceptores na tarefa de revisar prontuários, sob a perspectiva de uma abordagem centrada na pessoa, e as reflexôes desencadeadas acerca da preceptoria neste cenário de ensino-aprendizagem.

\section{Descrição da experiência}

O presente trabalho foi realizado como parte das exigências do Curso de Formação Pedagógica para a Prática de Preceptoria, promovido pelo Hospital Universitário Pedro Ernesto, no período de maio de 2010 a abril de 2011. Trata-se da construçáo de um instrumento de ensino-aprendizagem dirigido ao curso médico, que, para sua legitimação e uso no serviço, foi submetido à avaliação por pares.

A construção do roteiro para orientar a revisão do prontuário foi feita com base nos conteúdos trabalhados nas disciplinas de Medicina Integral, oferecidas aos estudantes na graduação, e em informaçóes consideradas relevantes para o acompanhamento médico na APS.,

Depois da apreciação da primeira versão do roteiro por três preceptores do internato, modificaçóes foram feitas para aprimoramento da forma e do conteúdo, que ao final ficou constituído de questóes fechadas e abertas, com os seguintes campos (Ver Anexo 1):

1) Identificação do paciente, motivo e data da primeira consulta no AMI.

2) Informaçóes relativas a aspectos relacionais e da subjetividade do paciente (lista de problemas, eventos da vida, aspectos familiares e psicossociais).

3) Informaçóes objetivas (dados relevantes do exame físico e dos exames complementares).

4) Avaliação das necessidades de saúde do paciente, do plano terapêutico, da qualidade dos registros e da longitudinalidade do cuidado.

5) Súmula diagnóstica e terapêutica, proposições para a melhoria do cuidado.

6) Avaliação das necessidades de aprendizagem pelo interno.

Um prontuário foi escolhido aleatoriamente, copiado e distribuído a seis preceptores voluntários, juntamente com uma cópia impressa do roteiro, que também foi disponibilizado por meio digital aos interessados, ficando a critério de cada um a escolha quanto à forma de preenchimento.

A revisão do prontuário foi também feita por uma das autoras, com o intuito de compreender eventuais dúvidas na utilizaçấo do roteiro pelos demais preceptores. Os roteiros preenchidos foram analisados, atentando-se para a frequência de cada resposta encontrada, considerando-se como concordância na resposta quando todos os preceptores davam a mesma resposta à pergunta feita. Os resultados foram então sistematizados e apresentados aos preceptores em reunião para discussão 
e reflexão, visando avaliar a pertinência do uso do roteiro no AMI como estratégia de ensino-aprendizagem para internos e preceptores.

O preenchimento do roteiro foi feito em papel por cinco preceptores, e por meio digital por um preceptor, sendo que esta forma permitiu melhor organização dos dados referentes aos resultados de exame físico e exames complementares. Dados elementares como os de identificaçáo do paciente estavam ausentes, o que vai ao encontro dos resultados apresentados no estudo de Silva e Tavares-Neto9 sobre prontuários de hospitais de ensino do Brasil.

Quanto ao conteúdo dos registros médicos, houve consenso entre os preceptores de que eram essencialmente centrados na doença. Muitas informaçôes importantes para a compreensão do processo de saúde-adoecimento dos usuários estavam ausentes ou desatualizadas, não havendo informaçôes sobre aspectos relacionados à família, aos eventos importantes da vida ou mesmo sobre as condiçôes de vida do usuário. Apesar de serem informaçóes valorizadas no serviço, nem sempre são registradas no prontuário, como evidenciado por Sarti et al. ${ }^{8} \mathrm{em}$ investigação realizada com médicos do AMI.

Observou-se concordância em $60 \%$ das respostas às perguntas fechadas. Houve dificuldade em respostas do tipo "sim ou não" em algumas perguntas, nas quais seria mais adequado incluir respostas intermediárias do tipo "algumas vezes", "nem sempre" ou "na maioria das vezes".

A concordância nas respostas às questôes abertas foi menor, pela compreensão diferenciada sobre fatores de risco ou proteção, vulnerabilidade e necessidades não percebidas pelo usuário em relação aos registros analisados, o que pode ser um reflexo da heterogeneidade da formação profissional dos preceptores do serviço.

Todos os preceptores fizeram observaçóes em relação à desorganização dos documentos contidos no prontuário (folhas de evoluçáo, resultados de exames ou de parecer). Isto se refletiu no tempo necessário para realizar a revisão do prontuário, já que demandou a organização e ordenação prévia dos registros para encontrar informaçóes relevantes. Considerando-se que alguns pacientes são acompanhados há muitos anos no serviço, é de se imaginar o esforço despendido pelos estudantes a fim de obterem dados mínimos sobre os pacientes antes do atendimento, bem como a repercussão disso sobre o cuidado, sobre o fluxo das consultas no ambulatório e sobre os gastos com exames solicitados indevidamente quando a revisão do prontuário não é realizada.

Os registros do prontuário utilizado para validação do roteiro, apesar de legíveis, continham, na maioria das vezes, a identificação do interno ou residente, mas não do preceptor, embora este pudesse ser identificado por meio de pedidos de exames contidos no prontuário.

Os preceptores avaliaram que o uso do roteiro trouxe ganhos para a sua prática de preceptoria, na medida em que puderam refletir sobre a importância da educação permanente para o desenvolvimento de competências médicas e pedagógicas para a atuação neste cenário de ensino-aprendizagem, assim como sobre a necessidade de ficarem mais atentos aos registros feitos pelos alunos.

Além disso, esta avaliação tornou evidente a necessidade de elaboração de outras fichas para compor o prontuário, com destaque para a identificação dos usuários, a ser atualizada pelos internos anualmente quando no início do rodízio, e outras que orientem o registro de uma abordagem centrada nos pacientes e não na doença, que possam contribuir como indutores para mudanças das práticas assistenciais dos médicos em formação, quer seus registros sejam em papel ou em meio eletrônico. ${ }^{10}$

\section{Discussão}

O termo prontuário vem do latim promptuarium, "[...] lugar em que se guardam as coisas que devem estar à mão, despensa, armário [...]" 11 e refere-se ao conjunto de documentos relativos à assistência prestada a um paciente. ${ }^{12}$ Os dados aí constantes pertencem ao paciente e à instituição que o assiste, sendo a denominação correta: prontuário médico do paciente. ${ }^{12}$ Trata-se de documento valioso não somente para o paciente, mas para o médico que o assiste e para as instituiçóes de saúde, para o ensino, a pesquisa, a administração dos serviços públicos de saúde e para a avaliação da qualidade, além de servir como instrumento de defesa legal. ${ }^{13}$ Todos os profissionais de saúde devem manter registros completos e precisos e serem responsáveis pelo conteúdo por eles gerado, sendo obrigatória, nos registros em papel, a legibilidade da letra do profissional que atende o paciente, bem como a assinatura e o respectivo número do registro no Conselho Regional de Medicina (CRM) nos casos de atendimento por médicos. ${ }^{13}$ 
Os registros das consultas neste cenário ambulatorial - de ensino-aprendizagem de práticas centradas no paciente - ganham uma relevância ainda maior do que nos serviços de saúde em geral, pois, além de serem essenciais para a continuidade das informaçóes sobre os parâmetros biomédicos entre os internos ano após ano, os registros precisam trazer indicativos sobre os encontros e vínculos estabelecidos entre os internos e os pacientes, de modo que haja a continuidade na confiança e corresponsabilização pelo projeto terapêutico em curso.

Entretanto, partindo-se do princípio de que "vale o que está escrito", e que a redação no prontuário é uma representação do contato estabelecido entre o interno e seu paciente, inferimos que os relatos feitos no prontuário analisado foram regidos pelo paradigma biomédico, que, tendo emergido no século XIX com a medicina científica, ${ }^{3}$ ainda hoje estrutura a maior parte do currículo das faculdades de medicina ditas tradicionais.

A internalização desta estrutura (modelo biomédico) nos sujeitos (estudantes de medicina), por meio da educação médica hegemônica, acaba se constituindo em um conjunto estável de disposiçôes estruturadas (valores) que, por sua vez, estruturam as práticas (diagnóstico de doenças e sua cura) e suas representaçôes (registros médicos no prontuário). ${ }^{14}$

É importante frisar que este processo se dá tanto pelo aprendizado direto, por meio de recursos didáticos diversos, como pelo aprendizado indireto, no qual as atitudes, valores e padrôes de comportamento são adquiridos como subprodutos do envolvimento contínuo com professores, colegas, pacientes e membros das equipes de saúde, que constituem a escola médica como uma organização social. ${ }^{15}$

Observa-se o desafio que é avaliar, orientar e operacionalizar a produção de registros médicos em atividades complexas de ensino-aprendizagem nos cenários reais de prática clínica. Esta complexidade é maior quando há a perspectiva de desenvolvimento de competências voltadas para uma prática centrada na pessoa, na família e na comunidade. ${ }^{3}$

A narrativa médica tradicional caracteriza-se por um processo descritivo de objetos (sinais, sintomas, exames, fármacos) encontrados no caso de um paciente. Desse modo, seu objetivo é traduzir narrativas dos pacientes em objetos/fatos médicos e, a partir deles, rotular, quantificar e classificar tais narrativas em doenças e tratamentos. Neste movimento, os registros abstraem os significados dos objetos da experiência do paciente em relação ao seu adoecimento. Os contextos familiar e sociocultural são incluídos como forma de explicar e justificar diagnósticos, mas não como elementos na construção da experiência do adoecer e da busca pelo cuidado. ${ }^{16}$

Portanto, as narrativas médicas e seus textos se caracterizam como instrumentos de informação e sistematização dos objetos médicos (sinais, sintomas, exames, fármacos) e não de reflexão sobre a experiência da pessoa, da família e da comunidade. Transcender estes limites implica uma escrita reflexiva, que, ao se aproximar da experiência de quem sofre, produz novos significados para o médico e para o paciente.

A revisão do prontuário pelo interno deve ser orientada pelo preceptor, objetivando levar o primeiro a refletir sobre a elaboração de textos que expressem a vivência das pessoas atendidas e a relação estabelecida com elas, para dar materialidade e relevância a aspectos menosprezados em uma prática ambulatorial centrada na doença.

\section{Conclusões}

O uso do roteiro para orientar a revisão dos prontuários foi aprovado pelos preceptores do ambulatório de Medicina Integral como estratégia de ensino-aprendizagem para os internos quanto à abordagem centrada na pessoa. Compreendeu-se que as revisôes do prontuário nestes moldes fornecem material adequado para subsidiar as sessóes clínicas do serviço e a educaçáo permanente sobre a prática da preceptoria, de modo a qualificar tanto o ensino médico como a assistência oferecida aos usuários.

A construção de registros médicos que se harmonizem com o ensino da prática centrada na pessoa é um desafio a ser enfrentado e pode se tornar ainda maior no momento em que os prontuários eletrônicos se tornem a interface entre as narrativas dos pacientes e os textos médicos. 


\section{Referências}

1. Stewart M, Weston WW, McWhinney IR, McWilliam CL, Freeman TR, Meredith L, et al. Medicina centrada na pessoa: transformando o método clínico: tradução: Anelise Teixeira Burmeister. Porto Alegre: Artmed; 2010.

2. Platt FW, Gaspar DL, Coulehan JL, Fox L, Adler AJ, Weston WW, et al. Tell Me about Yourself. The Patient-Centered Interview. Ann Intern Med. June 2001; 134 (11): 1079-85. PMid:11388827. http://dx.doi.org/10.7326/0003-4819-134-11-200106050-00020

3. McWhinney IR, Freeman T. Manual de Medicina de família e Comunidade. 3. ed. Porto Alegre: Artmed; 2010.

4. Brasil. Resolução do Conselho Nacional de Educação. Câmara de Educação Superior n 4, de 7 de novembro de 2001. [acesso em 2009 feb 28]. Disponível em: http://portal.mec.gov.br/cne/arquivos/pdf/CES04.pdf.

5. Afonso DH, Itikawa FA. Resumo de Relatos; II Mostra Nacional de Medicina de Família e Comunidade; $44^{\circ}$ Congresso Brasileiro de Educação Médica (COBEM), Gramado, 2006. Rev Bras Med Fam Comunidade. 2006; Supl.1: 67.

6. Noronha JC, Oliveira JÁ, Rodrigues RD, Landmann J. Transformações de um ambulatório de medicina integral com vistas a um programa de atenção médica primária: A experiência do Hospital de Clínicas da Universidade do Estado do Rio de Janeiro, Brasil. Rev Saude Publica. $2007 ; 11: 429-43$. http://dx.doi.org/10.1590/S0034-89101977000400001

7. Moss, MB. Meu primeiro ambulatório. In: Vasconcelos EM, Frota LH, Simon E, organizers. Perplexidade na universidade: vivências nos cursos de saúde. São Paulo: Editora Hucitec. p. 170-172.

8. Sarti TD, Anderson MIP, Marto RH. Registros clínicos e atenção primária: reflexões frente à abordagem biopsicossocial. APS. 2008; 11(4): 413-20. Disponível em: http://www.aps.ufjf.br/index.php/aps/article/viewArticle/275

9. Silva FG, Tavares-Neto J. Avaliação dos Prontuários Médicos de Hospitais de Ensino do Brasil. Rev Bras Educ Med. 2007; 31(2): 113-26. http:// dx.doi.org/10.1590/S0100-55022007000200002

10. Roman, AC. Informatização do registro clínico essencial para a atenção primária à saúde: um instrumento de apoio às equipes da estratégia saúde da família [tese]. São Paulo: Faculdade de Medicina; 2009 [acesso em 2012 jul 15]. Disponível em: http://www.teses.usp.br/teses/disponiveis/5/5144/ tde-28082009-095729/

11. Dicionário Eletrônico Houaiss CD-ROM v. 1.0 - dezembro de 2001.

12. Conselho Regional de Medicina do Distrito Federal. Prontuário médico do paciente: guia para uso prático / Conselho Regional de Medicina do Distrito Federal - Brasília: Conselho Regional de Medicina; 2006. 94 p. [acesso em 2012 jul 15]. Disponível em: http://www.periciamedicadf.com. br/publicacoes/prontuario_medico_paciente.pdf

13. Conselho Federal de Medicina. Resolução CFM n 1.638/2002 (Publicada no D.O.U. de 9 de agosto de 2002, Seção I, p.184-5). Define prontuário médico e torna obrigatória a criação da Comissão de Revisão de Prontuários nas instituições de saúde. [acesso em 2012 jul 15]. Disponível em: http://www.portalmedico.org.br/resolucoes/cfm/2002/1638_2002.htm

14. Nogueira MA, Nogueira CMM. Entre o subjetivismo e o objetivismo: em busca de uma superação. In: Horizonte: Autentica; 2009. cap. 1.

15. Rego S. Educação médica: histórias e questões. In: Rio de Janeiro: Editora Fiocruz, 2003. cap. 1. A formação ética dos médicos: saindo da adolescência com a vida (dos outros) na mão.

16. Greenhalgh TH, Hurwitz B. Narrative based medicine in an evidence based world. In: clinical practice. London: BMJ books; 1999. cap 24. Narrative based medicine: Dialogue and discourse in 
Anexo 1. Roteiro para orientar a revisão de prontuários.

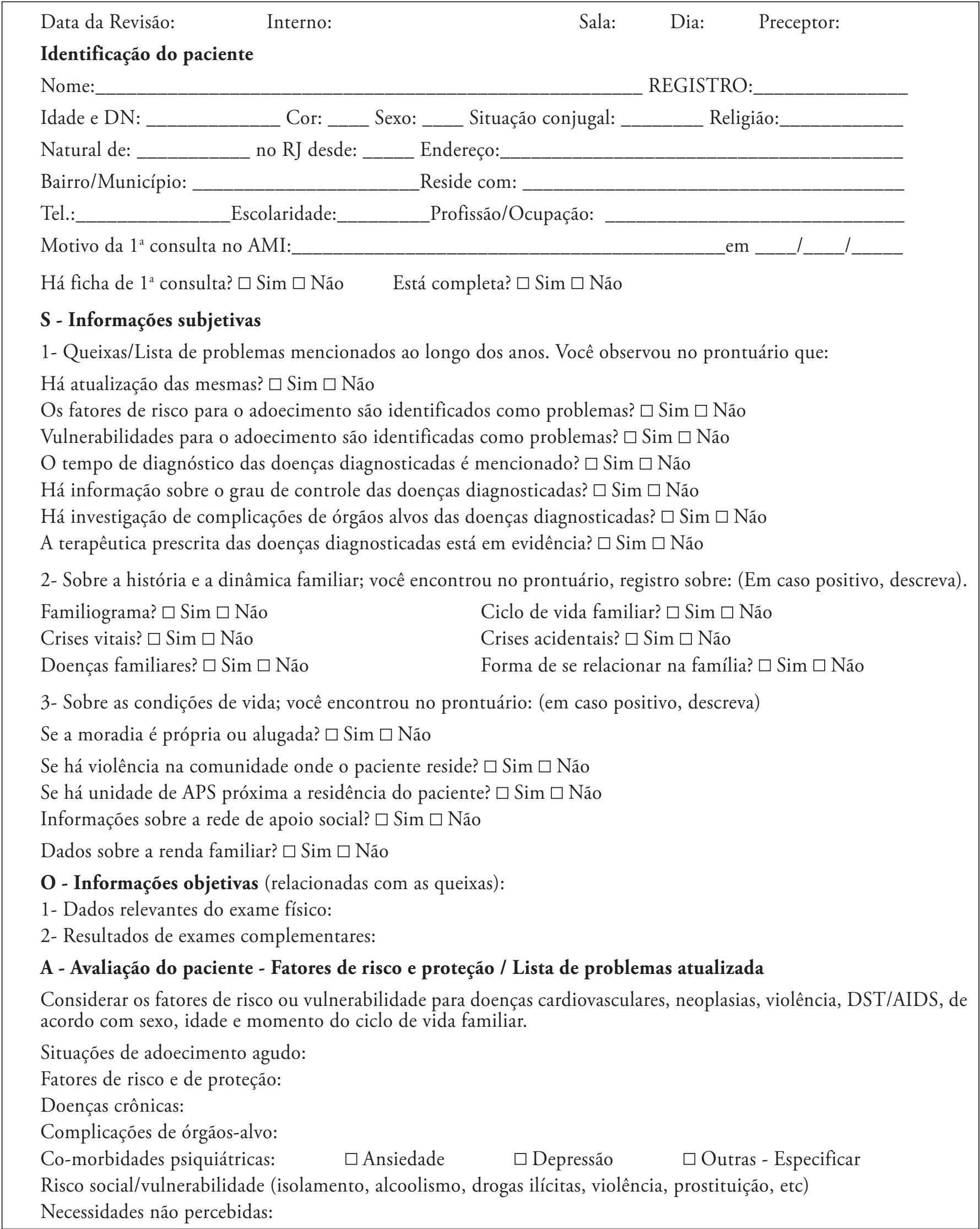


Anexo 1. Roteiro para orientar a revisão de prontuários.

\section{P - Plano Terapêutico}

1 - Orientações e aconselhamentos realizados. Mencione quais.

Educação em saúde nas consultas: $\square \operatorname{Sim} \square$ Não

Encaminhamento para grupos: $\square \operatorname{Sim} \square$ Não

Abordagem do contexto social:

Família: $\square$ Sim $\square$ Não $\quad$ Trabalho: $\square$ Sim $\square$ Não $\quad$ Escola: $\square$ Sim $\square$ Não

Comunidade: $\square \operatorname{Sim} \square$ Não

2 - Parecer solicitado? $\square$ Sim $\square$ Não

Qual? Respondidos? $\square$ Sim $\square$ Não

Interconsulta solicitada? $\square$ Sim $\square$ Não $\quad$ Qual? Conduta:

3 - Prescrição. Descreva e avalie.

Pertinentes? $\square$ Sim $\square$ Não $\quad$ Otimizadas? $\square$ Sim $\square$ Não $\quad$ Adesão? $\square$ Sim $\square$ Não

Efeitos colaterais? $\square$ Sim $\square$ Não

\section{Avaliação crítica dos registros}

Prontuário em ordem? $\square$ Sim $\square$ Não $\quad$ As informaçóes foram encontradas facilmente? $\square$ Sim $\square$ Não Registros legíveis? $\square$ Sim $\square$ Não

Há identificação do(s) responsável (eis) pelo cuidado ao longo do tempo? $\square \operatorname{Sim} \square$ Não

Alguma informação relevante sobre o paciente foi resgatada? $\square \operatorname{Sim} \square$ Não Qual?

\section{Avaliação da longitudinalidade do cuidado}

Há continuidade do cuidado de uma consulta para a outra e ao longo dos anos? $\square$ Sim $\square$ Não

É possível perceber uma coerência no cuidado? $\square$ Sim $\square$ Não

Foi observada uma abordagem biopsicossocial no cuidado? $\square \operatorname{Sim} \square$ Não

\section{Proposiçóes para a melhoria do cuidado}

Que informaçóes você não encontrou no prontuário que considera importante investigar e registrar?

Alguma sugestão para:

O plano terapêutico deste paciente? $\square$ Sim $\square$ Não Qual?

A organização do prontuário? $\square$ Sim $\square$ Não Qual?

A preceptoria? $\square$ Sim $\square$ Não Qual?

\section{Súmula diagnóstica e terapêutica}

Faça uma síntese da sua impressão sobre o caso:

\section{Avaliaçáo das necessidades de aprendizagem}

A partir da revisão do prontuário, identifique e justifique:

- Temas/assuntos que você precisa conhecer/estudar mais.

- Habilidades/atitudes que você precisa desenvolver/exercitar mais.

- Algum aprendizado/ganho para a sua formação a partir da revisão. 